\title{
First-Order Statistical Characteristics of Macrodiversity System with Three Microdiversity MRC Receivers in the Presence of $k-\mu$ Short-Term Fading and Gamma Long-Term Fading
}

\author{
Branimir Jaksic, ${ }^{1}$ Mihajlo Stefanovic, ${ }^{2}$ Danijela Aleksic, ${ }^{2}$ \\ Dragan Radenkovic, ${ }^{2}$ and Sinisa Minic ${ }^{3}$ \\ ${ }^{1}$ Faculty of Technical Sciences, University of Pristina, Kneza Milosa 7, 38220 Kosovska Mitrovica, Serbia \\ ${ }^{2}$ Faculty of Electrical Engineering, University of Nis, Aleksandra Medvedeva 14, 18000 Nis, Serbia \\ ${ }^{3}$ Teachers College, University of Pristina, Nemanjina, 38218 Leposavic, Serbia \\ Correspondence should be addressed to Branimir Jaksic; branimir.jaksic@pr.ac.rs
}

Received 25 July 2016; Accepted 1 November 2016

Academic Editor: George S. Tombras

Copyright (c) 2016 Branimir Jaksic et al. This is an open access article distributed under the Creative Commons Attribution License, which permits unrestricted use, distribution, and reproduction in any medium, provided the original work is properly cited.

Macrodiversity system with macrodiversity SC receiver and three microdiversity MRC (maximum ratio combining) receivers is considered. Independent $k-\mu$ short-term fading and correlated Gamma long-term fading are present at the inputs of microdiversity MRC receivers. For this model, the probability density function and the cumulative density function of microdiversity MRC receivers and macrodiversity SC receiver output signal envelopes are calculated. Influences of Gamma shadowing severity, $k-\mu$ multipath fading severity, Rician factor and correlation coefficient at probability density function, and cumulative density function of macrodiversity SC receiver output signal envelopes are graphically presented.

\section{Introduction}

Fading is a basic type of nuisance in wireless mobile telecommunication systems. Depending on propagation environment and different communications cases various types of fading can arise. Short-term fading is a result of signal propagation on multipath. The interaction of waves between transmitter and receiver (reflection, diffraction, and scattering) induces large numbers of sent copies signals on the input of receivers. Propagation environment can be linear and nonlinear. Nonlinear environment is defined as correlated surfaces in which dissipation field is not equal [1-3].

Long term fading arises because of shadow effect. Various objects create shadow effect in areas between transmitter and receiver. In most cases, long term fading is correlated. Changing of signal power due to the influence of shadow effect is slow in comparison to the signal envelope changing into short term fading. The signal envelope is variable due to short term fading, and the signal envelope power is variable due to the long term fading $[1,4]$.
The signal from the transmitter to the receiver can be propagated over one, two, or more clusters. Cluster is defined as waves which arrive at the inputs of receivers with approximately same delay. When the number of clusters increases, the fading severity decreases. Each cluster is formed by a pair of Gaussian components at the receiver $[2,5,6]$.

The statistical behavior of signal in such systems can be described by different distributions as Rayleigh, Rice, Nakagami- $m$, Weibull, or $k-\mu$. $k-\mu$ distribution can be used to describe the variation of the signal envelope in linear environments, with dominant component, several clusters in propagation environment, and equal components in quadrature of signal. $k-\mu$ distribution has two parameters. The parameter $k$ is Rician factor. Rician factor is defined as ratio of dominant components power and scattering components power. System performance is better for higher values of Rician factor. Rician factor increases as dominant components power increases or scattering components power decreases. The parameter $\mu$ is related to the number of clusters in propagation channels. The $k-\mu$ distribution is general distribution 
and several another distributions (Rician, Nakagami- $m$, and Rayleigh) can be obtained from $k-\mu$ distribution as special cases $[7,8]$.

Various diversity techniques to reduce the impact of short-term fading and long-term fading on the system performance can be used. The most commonly used are spatial diversion techniques. Spatial diversity techniques are implemented with multiple antennas mounted on the receiver. By using spatial diversity technique the reliability of the system and the channel capacity increase without increasing the transmitter power and frequency band expansion. There are several spatial diversity combining techniques that can be used to reduce the influence of fading and cochannel interference on system performances. The most commonly used diversity techniques are MRC (maximum ratio combining), EGC (equal gain combining), and SC (selection combining) $[2,7]$.

MRC diversity technique gives the best results. This technique effectively reduces the influence of $k$ - $\mu$ short term fading on the system performance and provides the greatest diversity gain. The ratio of signal power and noise at the output of the MRC receiver is equal to the sum of the ratio signal power and noise at its inputs. If noise power is the same in all diversity branches, then squared output signal is equal to the sum of the squares of the signal at its input. This method requires that the signals at the inputs are brought to the same phase. Because of that this method of combining is very complex and expensive for real implementation $[8,9]$.

There are more works in open technical literature considering second order statistics of diversity systems. In [10], average crossing rate and average fade duration of macrodiversity $\mathrm{SC}$ receiver with two microdiversity maximum ratio combining (MRC) receivers operating over Gamma shadowed Nakagami- $m$ multipath fading are calculated. Macrodiversity $S C$ receiver selects microdiversity receiver with higher input power to serve to user. In [11], average fade duration and level crossing rate of macrodiversity SC receiver with two microdiversity MRC receivers operating over Rician fading multipath environment are derived.

\section{System Model}

In this paper, macrodiversity system with macrodiversity SC (selection combining) receiver and three microdiversity MRC (maximum ratio combining) receivers is analyzed. At the inputs of microdiversity MRC receivers are independent $k-\mu$ short term fading and Gamma long term fading. Gamma long term fading is correlated. The correlation coefficient decreases with increasing distance between the antennas.

Microdiversity MRC receiver reduces $k-\mu$ short term fading effects and macrodiversity SC receivers reduce Gamma long term fading effects on system performances. Obtained macrosystem is predicted for one cell in the cellular mobile radio systems. Microdiversity receivers are placed on the base stations for mobile users of this cell. Macrodiversity systems used signals from several base stations placed in one cell (two or more) [2].

The system that is being considered is shown in Figure 1. The signals at the inputs and the outputs of the MRC receivers

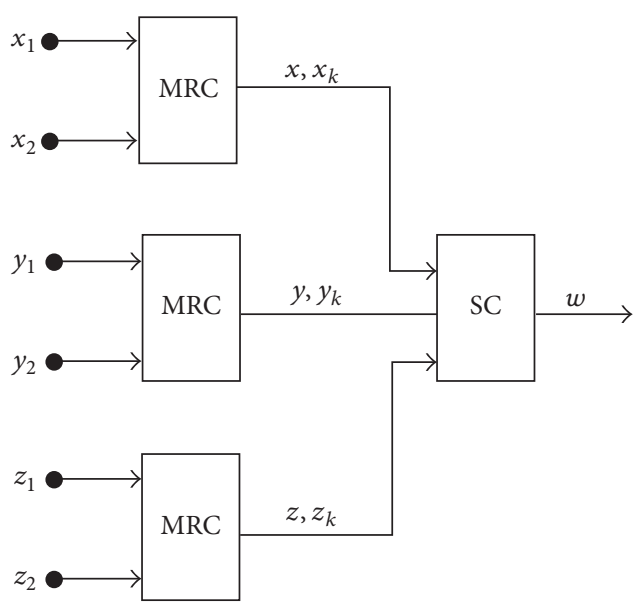

FIGURE 1: Macrodiversity system with three microdiversity MRC receivers and one macrodiversity $\mathrm{SC}$ receiver.

are denoted as in Figure 1. The signal at the output of macrodiversity system is denoted with $w$.

The square of the macrodiversity system output signal envelope is equal to sum of signal squares from its inputs. The macrodiversity SC receiver output signal envelope is equal to the microdiversity MRC receiver output signal envelope whose signal power at the input microdivesity $\mathrm{MRC}$ receiver is greater than the signal power at the input of the other two microdivesity MRC receivers.

Squared $k-\mu$ distribution is equal to sum of $2 \mu$ squares of Gaussian random independent with same variance variables. In this way we can determine the probability density function of $k-\mu$ distribution. With these probability density functions we can determine cumulative density function of $k-\mu$ random variables, characteristic function of the random variable $k-\mu$, and moments of the $k-\mu$ random variable.

Probability density function of macrodiversity SC receiver output signal envelope is equal to the probability density function of microdiversity MRC receiver output signal envelope with highest signal power at this input.

The cumulative density function is equal to integrating of probability density function. Thus can be calculated the cumulative density function of output signal envelope for the first, second, and third microdiversity MRC receiver. The cumulative density function of macrodiversity SC receiver output signal envelope can be determined by integrating the probability density function of macrodiversity SC receiver output signal envelope. By using the cumulative density function can be determined outage probability [3].

\section{Probability Density Function of Macrodiversity SC Receiver Output Signal Envelope}

Squared $k-\mu x_{1}$ signal is equal to

$$
x_{1}^{2}=x_{11}^{2}+x_{12}^{2}+\cdots+x_{12 \mu}^{2} .
$$


Squared $k-\mu x_{2}$ signal is equal to

$$
x_{2}^{2}=x_{21}^{2}+x_{22}^{2}+\cdots+x_{22 \mu}^{2} .
$$

The squared signal $x$ is equal to

$$
\begin{aligned}
x^{2} & =x_{1}^{2}+x_{2}^{2} \\
& =x_{11}^{2}+x_{12}^{2}+\cdots+x_{12 \mu}^{2}+x_{21}^{2}+x_{22}^{2}+\cdots+x_{22 \mu}^{2} \\
& =x_{k} .
\end{aligned}
$$

Random variable $x_{k}$ has a $\chi^{2}$ distribution:

$$
\begin{aligned}
& p_{x_{k}}\left(x_{k}\right) \\
& \quad=\frac{1}{2 \sigma^{2}}\left(\frac{x_{k}}{\lambda}\right)^{(2 \mu-1) / 2} e^{-\left(\lambda+x_{k}\right) / 2 \sigma^{2}} I_{2 \mu+1}\left(\frac{\lambda^{1 / 2} x_{k}^{1 / 2}}{\sigma^{2}}\right),
\end{aligned}
$$

where $I_{n}(\cdot)$ is modified Bessel function of the first kind, order $n$ and argument $x, \sigma$ signal variance, $\mu$ the number of clusters for the signal, and $\lambda$ the mean signal power.

Relations between $x_{k}$ and $x$ are

$$
\begin{gathered}
x^{2}=x_{k}, \\
x=x_{k}^{1 / 2} .
\end{gathered}
$$

Probability density function of microdiversity MRC receiver output signal envelope $x$ is

$$
p_{x}(x)=\frac{d x_{k}}{d x} p_{x_{k}}\left(x^{2}\right)
$$

where

$$
\frac{d x_{k}}{d x}=2 x
$$

By substituting (7) and (4) in (6) the development of Bessel functions is obtained:

$$
\begin{aligned}
p_{x}(x)= & \frac{1}{\sigma_{1}^{2} \lambda^{2 \mu-1}} \sum_{i_{1}=0}^{\infty}\left(\frac{\lambda^{1 / 2}}{2 \sigma_{1}^{2}}\right)^{2 i_{1}+2 \mu-1} \frac{1}{i_{1} ! \Gamma\left(i_{1}+2 \mu\right)} \\
& \cdot x^{2 i_{1}+4 \mu-1} e^{-\left(\lambda+x^{2}\right) / 2 \sigma_{1}^{2}} .
\end{aligned}
$$

One takes

$$
2 \sigma_{1}^{2}=\Omega_{1} .
$$

After substituting (9) in (8), probability density function of microdiversity MRC receiver output signal envelope becomes

$$
\begin{aligned}
p_{x}(x)= & \frac{2}{\Omega_{1} \lambda^{2 \mu-1}} \sum_{i_{1}=0}^{\infty} \lambda^{i_{1}+\mu-1 / 2} \Omega_{1}^{-i_{1}-\mu+1 / 2} \frac{1}{i_{1} ! \Gamma\left(i_{1}+2 \mu\right)} \\
& \cdot x^{2 i_{1}+4 \mu-1} e^{-\left(\lambda+x^{2}\right) / \Omega_{1}},
\end{aligned}
$$

where $\Omega_{1}$ means signal power.
In a similar way we can obtain the probability density function of microdiversity MRC receiver output signal envelope for second and third microdiversity MRC receiver:

$$
\begin{aligned}
& p_{y}(y)=\frac{2}{\Omega_{2} \lambda^{2 \mu-1}} \sum_{i_{2}=0}^{\infty} \lambda^{i_{2}+\mu-1 / 2} \Omega_{2}^{-i_{2}-\mu+1 / 2} \frac{1}{i_{2} ! \Gamma\left(i_{2}+2 \mu\right)} \\
& \cdot y^{2 i_{2}+4 \mu-1} e^{-\left(\lambda+y^{2}\right) / \Omega_{2}}, \\
& p_{z}(z)=\frac{2}{\Omega_{3} \lambda^{2 \mu-1}} \sum_{i_{3}=0}^{\infty} \lambda^{i_{3}+\mu-1 / 2} \Omega_{3}^{-i_{3}-\mu+1 / 2} \frac{1}{i_{3} ! \Gamma\left(i_{3}+2 \mu\right)} \\
& \cdot z^{2 i_{3}+4 \mu-1} e^{-\left(\lambda+z^{2}\right) / \Omega_{3}} .
\end{aligned}
$$

Signal envelopes powers at inputs in microdiversity receivers are correlated. Signal envelope powers $\Omega_{1}, \Omega_{2}$, and $\Omega_{3}$ follow Gamma distribution:

$$
\begin{aligned}
& p_{\Omega_{1} \Omega_{2} \Omega_{3}}\left(\Omega_{1} \Omega_{2} \Omega_{3}\right) \\
& =\frac{1}{\Gamma(c)\left(1-\rho^{2}\right) \rho^{c-1} \Omega_{0}^{c+2}}\left(\Omega_{1} \Omega_{3}\right)^{(c-1) / 2} \\
& \cdot e^{-\left(\Omega_{1}+\Omega_{3}+\Omega_{2}(1+\rho)\right) / \Omega_{0}(1-\rho)} I_{c-1}\left(\frac{2 \sqrt{\rho}}{\Omega_{0}(1-\rho)} \sqrt{\Omega_{1} \Omega_{2}}\right) \\
& \cdot I_{c-1}\left(\frac{2 \sqrt{\rho}}{\Omega_{0}(1-\rho)} \sqrt{\Omega_{2} \Omega_{3}}\right),
\end{aligned}
$$

where $I_{n}(\cdot)$ is modified Bessel function of the first kind, order $n$, and argument $x ; \Omega_{0}$ is mean square of signal power variation, $\rho$ correlation coefficient, and $c$ Gamma shadowing severity.

Probability density function of macrodiversity SC receiver output signal envelope $w$ is

$$
\begin{aligned}
& p_{w}(w)=\int_{0}^{\infty} d \Omega_{1} \int_{0}^{\Omega_{1}} d \Omega_{2} \\
& \cdot \int_{0}^{\Omega_{1}} d \Omega_{3} p_{x}\left(\frac{w}{\Omega_{1}}\right) p_{\Omega_{1} \Omega_{2} \Omega_{3}}\left(\Omega_{1} \Omega_{2} \Omega_{3}\right)+\int_{0}^{\infty} d \Omega_{2} \\
& \cdot \int_{0}^{\Omega_{2}} d \Omega_{1} \int_{0}^{\Omega_{2}} d \Omega_{3} p_{y}\left(\frac{w}{\Omega_{2}}\right) p_{\Omega_{1} \Omega_{2} \Omega_{3}}\left(\Omega_{1} \Omega_{2} \Omega_{3}\right) \\
& +\int_{0}^{\infty} d \Omega_{3} \int_{0}^{\Omega_{3}} d \Omega_{1} \\
& \cdot \int_{0}^{\Omega_{3}} d \Omega_{2} p_{z}\left(\frac{w}{\Omega_{3}}\right) p_{\Omega_{1} \Omega_{2} \Omega_{3}}\left(\Omega_{1} \Omega_{2} \Omega_{3}\right)=I_{1}+I_{2} \\
& +I_{3}=2 I_{1}+I_{2} .
\end{aligned}
$$

Functions $p_{x}\left(w / \Omega_{1}\right), p_{y}\left(w / \Omega_{2}\right)$, and $p_{z}\left(w / \Omega_{3}\right)$ are given in (10), (11), and (12), respectively. 
4

Journal of Electrical and Computer Engineering

Integral $I_{1}$ is equal to

$$
\begin{aligned}
I_{1} & =\frac{2}{\lambda^{2 \mu-1}} \frac{1}{\Gamma(c)\left(1-\rho^{2}\right) \rho^{c-1} \Omega_{0}^{c+2}} \\
& \cdot \sum_{i_{1}=0}^{\infty} \frac{1}{i_{1} ! \Gamma\left(i_{1}+2 \mu\right)} \lambda^{i_{1}+\mu-1 / 2} x^{2 i_{1}+4 \mu-1} \\
& \cdot \sum_{j_{1}=0}^{\infty}\left(\frac{\sqrt{\rho}}{\Omega_{0}(1-\rho)}\right)^{2 j_{1}+c-1} \frac{1}{j_{1} ! \Gamma\left(j_{1}+c\right)} \\
& \cdot \sum_{j_{2}=0}^{\infty}\left(\frac{\sqrt{\rho}}{\Omega_{0}(1-\rho)}\right)^{2 j_{2}+c-1} \frac{1}{j_{2} ! \Gamma\left(j_{2}+c\right)} \\
& \cdot \int_{0}^{\infty} d \Omega_{1} \Omega_{1}^{-1-i_{1}-\mu+1 / 2+j_{1}+c-1} e^{-\left(\lambda+x^{2}\right) / \Omega_{1}-\Omega_{1} / \Omega_{0}(1-\rho)} \\
& \cdot \int_{0}^{\Omega_{1}} d \Omega_{2} \Omega_{2}^{j_{1}+j_{2}+c-1} e^{-\Omega_{2}(1+\rho) / \Omega_{0}(1-\rho)} \\
& \cdot \int_{0}^{\Omega_{1}} d \Omega_{3} \Omega_{3}^{j_{2}+c-1} e^{-\Omega_{3} / \Omega_{0}(1-\rho)} .
\end{aligned}
$$

After using [12] for resolving the second and third integrals in (15), $I_{1}$ becomes

$$
\begin{aligned}
I_{1}= & \frac{2}{\lambda^{2 \mu-1}} \frac{1}{\Gamma(c)\left(1-\rho^{2}\right) \rho^{c-1} \Omega_{0}^{c+2}} \\
& \cdot \sum_{i_{1}=0}^{\infty} \frac{1}{i_{1} ! \Gamma\left(i_{1}+2 \mu\right)} \lambda^{i_{1}+\mu-1 / 2} x^{2 i_{1}+4 \mu-1} \\
& \cdot \sum_{j_{1}=0}^{\infty}\left(\frac{\sqrt{\rho}}{\Omega_{0}(1-\rho)}\right)^{2 j_{1}+c-1} \frac{1}{j_{1} ! \Gamma\left(j_{1}+c\right)} \\
& \cdot \sum_{j_{2}=0}^{\infty}\left(\frac{\sqrt{\rho}}{\Omega_{0}(1-\rho)}\right)^{2 j_{2}+c-1} \frac{1}{j_{2} ! \Gamma\left(j_{2}+c\right)} \\
& \cdot\left(\frac{\Omega_{0}(1-\rho)}{1+\rho}\right)^{j_{1}+j_{2}+c}\left(\Omega_{0}(1-\rho)\right)^{j_{2}+c} \\
& \cdot \int_{0}^{\infty} d \Omega_{1} \Omega_{1}^{-1-i_{1}-\mu+1 / 2+j_{1}+c-1} e^{-\left(\lambda+x^{2}\right) / \Omega_{1}-\Omega_{1} / \Omega_{0}(1-\rho)} \\
& \cdot \gamma\left(j_{1}+j_{2}+c, \frac{1+\rho}{\Omega_{0}(1-\rho)} \Omega_{1}\right) \\
& \cdot \gamma\left(j_{2}+c, \frac{1}{\Omega_{0}(1-\rho)} \Omega_{1}\right) \\
& (1)
\end{aligned}
$$

where $\gamma(\cdot)$ represents the lower incomplete Gamma function. After the developing Gamma function,

$$
\begin{aligned}
\gamma(n, x) & =\frac{1}{n} x^{n} e^{-x}{ }_{1} F_{1}(1, n+1, x) \\
& =\frac{1}{n} x^{n} e^{-x} \sum_{i=0}^{\infty} \frac{n !}{(n+i) !} x^{i},
\end{aligned}
$$

$I_{1}$ becomes

$$
\begin{aligned}
& I_{1}=\frac{2}{\lambda^{2 \mu-1}} \frac{1}{\Gamma(c)\left(1-\rho^{2}\right) \rho^{c-1} \Omega_{0}^{c+2}} \\
& \cdot \sum_{i_{1}=0}^{\infty} \frac{1}{i_{1} ! \Gamma\left(i_{1}+2 \mu\right)} \lambda^{i_{1}+\mu-1 / 2} x^{2 i_{1}+4 \mu-1} \\
& \cdot \sum_{j_{1}=0}^{\infty}\left(\frac{\sqrt{\rho}}{\Omega_{0}(1-\rho)}\right)^{2 j_{1}+c-1} \frac{1}{j_{1} ! \Gamma\left(j_{1}+c\right)} \\
& \cdot \sum_{j_{2}=0}^{\infty}\left(\frac{\sqrt{\rho}}{\Omega_{0}(1-\rho)}\right)^{2 j_{2}+c-1} \frac{1}{j_{2} ! \Gamma\left(j_{2}+c\right)} \\
& \cdot\left(\frac{\Omega_{0}(1-\rho)}{1+\rho}\right)^{j_{1}+j_{2}+c}\left(\Omega_{0}(1-\rho)\right)^{j_{2}+c} \\
& \cdot \int_{0}^{\infty} d \Omega_{1} \Omega_{1}^{-1-i_{1}-\mu+1 / 2+j_{1}+c-1} e^{-\left(\lambda+x^{2}\right) / \Omega_{1}-\Omega_{1} / \Omega_{0}(1-\rho)} \\
& \frac{1}{j_{1}+j_{2}+c}\left(\frac{(1+\rho) \Omega_{1}}{\Omega_{0}(1-\rho)}\right)^{j_{1}+j_{2}+c} \\
& \cdot e^{-(1+\rho) \Omega_{1} / \Omega_{0}(1-\rho)} \sum_{j_{3}=0}^{\infty} \frac{\left(j_{1}+j_{2}+c\right) !}{\left(j_{1}+j_{2}+c+j_{3}\right) !} \\
& \cdot\left(\frac{(1+\rho) \Omega_{1}}{\Omega_{0}(1-\rho)}\right)^{j_{3}} \frac{1}{j_{2}+c}\left(\frac{\Omega_{1}}{\Omega_{0}(1-\rho)}\right)^{j_{2}+c} \\
& \cdot e^{-\Omega_{1} / \Omega_{0}(1-\rho)} \sum_{j_{4}=0}^{\infty} \frac{\left(j_{2}+c\right) !}{\left(j_{2}+c+j_{4}\right) !}\left(\frac{\Omega_{1}}{\Omega_{0}(1-\rho)}\right)^{j_{4}} \text {. }
\end{aligned}
$$

By using [12] the following is obtained:

$$
\begin{aligned}
I_{1}= & \frac{2}{\lambda^{2 \mu-1}} \frac{1}{\Gamma(c)\left(1-\rho^{2}\right) \rho^{c-1} \Omega_{0}^{c+2}} \sum_{i_{1}=0}^{\infty} \frac{1}{i_{1} ! \Gamma\left(i_{1}+2 \mu\right)} \\
& \cdot \lambda^{i_{1}+\mu-1 / 2} x^{2 i_{1}+4 \mu-1} \sum_{j_{1}=0}^{\infty}\left(\frac{\sqrt{\rho}}{\Omega_{0}(1-\rho)}\right)^{2 j_{1}+c-1} \frac{1}{j_{1} ! \Gamma\left(j_{1}+c\right)} \\
& \cdot \sum_{j_{2}=0}^{\infty}\left(\frac{\sqrt{\rho}}{\Omega_{0}(1-\rho)}\right)^{2 j_{2}+c-1} \\
& \cdot \frac{1}{j_{2} ! \Gamma\left(j_{2}+c\right)}\left(\frac{\Omega_{0}(1-\rho)}{1+\rho}\right)^{j_{1}+j_{2}+c} \\
& \cdot \frac{1}{j_{1}+j_{2}+c}\left(\frac{(1+\rho)}{\Omega_{0}(1-\rho)}\right)^{j_{1}+j_{2}+c} \\
& \cdot \sum_{j_{3}=0}^{\infty} \frac{\left(j_{1}+j_{2}+c\right) !}{\left(j_{1}+j_{2}+c+j_{3}\right) !}\left(\frac{(1+\rho))^{j_{2}+c}}{\Omega_{0}(1-\rho)}\right)^{j_{3}} \frac{1}{j_{2}+c} \\
& \cdot \frac{1}{\left(\Omega_{0}(1-\rho)\right)^{j_{2}+c}} \sum_{j_{4}=0}^{\infty} \frac{\left(j_{2}+c\right) !}{\left(j_{2}+c+j_{4}\right) !} \frac{1}{\left(\Omega_{0}(1-\rho)\right)^{j_{4}}} \\
& \cdot K_{-1 / 2-i_{1}-\mu+2 j_{1}+2 j_{2}+j_{3}+j_{4}+3 c}\left(2 \sqrt{\frac{\left(\lambda+x^{2}\right)(3+\rho)}{\Omega_{0}(1-\rho)}}\right), \\
& \cdot\left(\frac{\left.\lambda+x^{2}\right) \Omega_{0}(1-\rho)}{(3+\rho)}\right)
\end{aligned}
$$


where $K_{n}(x)$ is modified Bessel function of the second kind, order $n$, and argument $x$.

Integral $I_{2}$ is equal to

$$
\begin{aligned}
I_{2}= & \frac{2}{\lambda^{2 \mu-1}} \frac{1}{\Gamma(c)\left(1-\rho^{2}\right) \rho^{c-1} \Omega_{0}^{c+2}} \\
& \cdot \sum_{i_{2}=0}^{\infty} \frac{1}{i_{2} ! \Gamma\left(i_{2}+2 \mu\right)} \lambda^{i_{2}+\mu-1 / 2} y^{2 i_{2}+4 \mu-1} \\
& \cdot \sum_{j_{1}=0}^{\infty}\left(\frac{\sqrt{\rho}}{\Omega_{0}(1-\rho)}\right)^{2 j_{1}+c-1} \frac{1}{j_{1} ! \Gamma\left(j_{1}+c\right)} \\
& \cdot \sum_{j_{2}=0}^{\infty}\left(\frac{\sqrt{\rho}}{\Omega_{0}(1-\rho)}\right)^{2 j_{2}+c-1} \frac{1}{j_{2} ! \Gamma\left(j_{2}+c\right)} \\
& \cdot \int_{0}^{\infty} d \Omega_{2} \Omega_{2}^{-1 / 2-i_{2}-\mu+j_{1}+c-1} e^{-\left(\lambda+y^{2}\right) / \Omega_{2}-\Omega_{2}(1+\rho) / \Omega_{0}(1-\rho)} \\
& \cdot \int_{0}^{\Omega_{2}} d \Omega_{1} \Omega_{1}^{j_{1}+c-1} e^{-\Omega_{1} / \Omega_{0}(1-\rho)} \\
& \cdot \int_{0}^{\Omega_{2}} d \Omega_{3} \Omega_{3}^{j_{2}+c-1} e^{-\Omega_{3} / \Omega_{0}(1-\rho)} .
\end{aligned}
$$

After the using procedure for solving $I_{1}, I_{2}$ becomes

$$
\begin{aligned}
I_{2}= & \frac{2}{\lambda^{2 \mu-1}} \frac{1}{\Gamma(c)\left(1-\rho^{2}\right) \rho^{c-1} \Omega_{0}^{c+2}} \\
& \cdot \sum_{i_{2}=0}^{\infty} \frac{1}{i_{2} ! \Gamma\left(i_{2}+2 \mu\right)} \lambda^{i_{2}+\mu-1 / 2} y^{2 i_{2}+4 \mu-1} \\
& \cdot \sum_{j_{1}=0}^{\infty}\left(\frac{\sqrt{\rho}}{\Omega_{0}(1-\rho)}\right)^{2 j_{1}+c-1} \frac{1}{j_{1} ! \Gamma\left(j_{1}+c\right)} \\
& \cdot \sum_{j_{2}=0}^{\infty}\left(\frac{\sqrt{\rho}}{\Omega_{0}(1-\rho)}\right)^{2 j_{2}+c-1} \frac{\left(\Omega_{0}(1-\rho)\right)^{j_{1}+j_{2}+2 c}}{j_{2} ! \Gamma\left(j_{2}+c\right)} \frac{1}{j_{1}+c} \\
& \cdot \frac{1}{\left(\Omega_{0}(1-\rho)\right)^{j_{1}+c}} \sum_{j_{3}=0}^{\infty} \frac{\left(j_{1}+c\right) !}{\left(j_{1}+c+j_{3}\right) !} \frac{1}{\left(\Omega_{0}(1-\rho)\right)^{j_{3}}} \frac{1}{j_{2}+c} \\
& \cdot \frac{1}{\left(\Omega_{0}(1-\rho)\right)^{j_{2}+c}} \sum_{j_{4}=0}^{\infty} \frac{\left(j_{2}+c\right) !}{\left(j_{2}+c+j_{4}\right) !} \frac{1}{\left(\Omega_{0}(1-\rho)\right)^{j_{4}}} \\
& \cdot\left(\frac{\left(\lambda+y^{2}\right) \Omega_{0}(1-\rho)}{(3+\rho)}\right)^{-1 / 4-i_{2} / 2-\mu / 2+j_{1}+j_{2} / 2+j_{3} / 2+j_{4} / 2+3 c / 2} \\
& \cdot K_{-1 / 2-i_{2}-\mu+2 j_{1}+j_{2}+j_{3}+j_{4}+3 c}\left(2 \sqrt{\frac{\left(\lambda+y^{2}\right)(3+\rho)}{\Omega_{0}(1-\rho)}}\right) .
\end{aligned}
$$

After replacing the integrals $I_{1}$ and $I_{2}$ in (14), we obtain an expression for PDF of macrodiversity SC receiver output signal envelope:

$$
\begin{aligned}
p= & \frac{8}{\lambda^{2 \mu-1}} \frac{1}{\Gamma(c)\left(1-\rho^{2}\right) \rho^{c-1} \Omega_{0}^{c+2}} \sum_{i_{1}=0}^{\infty} \frac{\lambda^{i_{1}+\mu-1 / 2}}{i_{1} ! \Gamma\left(i_{1}+2 \mu\right)} \\
& \cdot \sum_{j_{1}=0}^{\infty}\left(\frac{\sqrt{\rho}}{\Omega_{0}(1-\rho)}\right)^{2 j_{1}+c-1} \frac{1}{j_{1} ! \Gamma\left(j_{1}+c\right)}
\end{aligned}
$$

$$
\begin{aligned}
& \cdot \sum_{j_{2}=0}^{\infty}\left(\frac{\sqrt{\rho}}{\Omega_{0}(1-\rho)}\right)^{2 j_{2}+c-1} \frac{1}{j_{2} ! \Gamma\left(j_{2}+c\right)} \\
& \cdot x^{2 i_{1}+4 \mu-1}\left(\frac{\Omega_{0}(1-\rho)}{1+\rho}\right)^{j_{2}+c} \frac{1}{j_{1}+j_{2}+c} \\
& \cdot \sum_{j_{3}=0}^{\infty} \frac{\left(j_{1}+j_{2}+c\right) !}{\left(j_{1}+j_{2}+c+j_{3}\right) !}\left(\frac{(1+\rho)}{\Omega_{0}(1-\rho)}\right)^{j_{3}} \frac{1}{j_{2}+c} \\
& \cdot \frac{1}{\left(\Omega_{0}(1-\rho)\right)^{j_{2}+c}} \sum_{j_{4}=0}^{\infty} \frac{\left(j_{2}+c\right) !}{\left(j_{2}+c+j_{4}\right) !} \frac{1}{\left(\Omega_{0}(1-\rho)\right)^{j_{4}}} \\
& \left.\cdot \frac{\left(\lambda+x^{2}\right) \Omega_{0}(1-\rho)}{(3+\rho)}\right)^{-1 / 4-i_{1} / 2-\mu / 2+j_{1}+j_{2}+j_{3} / 2+j_{3} / 2+3 c / 2} \\
& \left.\cdot \frac{\left(\lambda+y^{2}\right) \Omega_{0}(1-\rho)}{(3+\rho)}\right)^{-1 / 4-i_{1} / 2-\mu / 2+j_{1}+j_{2} / 2+j_{3} / 2+j_{4} / 2+3 c / 2} \\
& \cdot \sum_{j_{4}=0}^{\infty} \frac{\left(j_{2}+c\right) !}{\left(j_{2}+c+j_{4}\right) !} \frac{1}{\left(\Omega_{0}(1-\rho)\right)^{j_{4}}} \\
& +\sum_{-1 / 2-i_{1}-\mu+2 j_{1}+2 j_{2}+j_{3}+j_{4}+3 c} \frac{\left(j_{1}+c\right) !}{\left(j_{1}+c+j_{3}\right) !} \frac{1}{\left(j_{3}+j_{4}+3 c\right.}\left(\frac{\left(\lambda+x^{2}\right)(3+\rho)}{\Omega_{0}(1-\rho)}\right) \\
& +y^{2 i_{1}+4 \mu-1} \frac{\left(\Omega_{0}(1-\rho)\right)^{j_{2}+j_{3}+c}}{j_{2}+c}
\end{aligned}
$$

\section{Cumulative Density Function of Macrodiversity SC Receiver Output Signal Envelope}

Probability density function of microdiversity MRC receiver output signal envelope $x$ is

$$
\begin{gathered}
F_{x}(x)=\int_{0}^{x} d t p_{x}(t)=\frac{1}{\Omega_{1} \lambda^{2 \mu-1}} e^{-\lambda / \Omega_{1}} \sum_{i_{1}=0}^{\infty} \lambda^{i_{1}+\mu-1 / 2} \\
\cdot \Omega_{1}^{-i_{1}-\mu+1 / 2} \frac{1}{i_{1} ! \Gamma\left(i_{1}+2 \mu\right)} \Omega_{1}^{i_{1}+2 \mu} \gamma\left(i_{1}+2 \mu, \frac{x^{2}}{\Omega_{1}}\right),
\end{gathered}
$$

where $p_{x}(t)$ is probability density function given by (10). 
In a similar way we can obtain the cumulative density function of microdiversity MRC receiver output signal envelope for second and third microdiversity MRC receiver:

$$
\begin{gathered}
F_{y}(y)=\frac{1}{\Omega_{2} \lambda^{2 \mu-1}} e^{-\lambda / \Omega_{2}} \sum_{i_{2}=0}^{\infty} \lambda^{i_{2}+\mu-1 / 2} \Omega_{2}^{-i_{2}-\mu+1 / 2} \\
\cdot \frac{1}{i_{2} ! \Gamma\left(i_{2}+2 \mu\right)} \Omega_{2}^{i_{2}+2 \mu} \gamma\left(i_{2}+2 \mu, \frac{y^{2}}{\Omega_{2}}\right) \\
F_{z}(z)=\frac{1}{\Omega_{3} \lambda^{2 \mu-1}} e^{-\lambda / \Omega_{3}} \sum_{i_{3}=0}^{\infty} \lambda^{i_{3}+\mu-1 / 2} \Omega_{1}^{-i_{3}-\mu+1 / 2} \\
\cdot \frac{1}{i_{3} ! \Gamma\left(i_{3}+2 \mu\right)} \Omega_{1}^{i_{3}+2 \mu} \gamma\left(i_{3}+2 \mu, \frac{z^{2}}{\Omega_{3}}\right) .
\end{gathered}
$$

Cumulative density function of macrodiversity SC receiver output signal envelope $w$ is

$$
\begin{aligned}
& F_{w}(w)=\int_{0}^{\infty} d \Omega_{1} \int_{0}^{\Omega_{1}} d \Omega_{2} \\
& \cdot \int_{0}^{\Omega_{1}} d \Omega_{3} F_{x}\left(\frac{w}{\Omega_{1}}\right) p_{\Omega_{1} \Omega_{2} \Omega_{3}}\left(\Omega_{1} \Omega_{2} \Omega_{3}\right) \\
& \quad+\int_{0}^{\infty} d \Omega_{2} \int_{0}^{\Omega_{2}} d \Omega_{1} \\
& \cdot \int_{0}^{\Omega_{2}} d \Omega_{3} F_{y}\left(\frac{w}{\Omega_{2}}\right) p_{\Omega_{1} \Omega_{2} \Omega_{3}}\left(\Omega_{1} \Omega_{2} \Omega_{3}\right) \\
& \quad+\int_{0}^{\infty} d \Omega_{3} \int_{0}^{\Omega_{3}} d \Omega_{1} \\
& \cdot \int_{0}^{\Omega_{3}} d \Omega_{2} F_{z}\left(\frac{w}{\Omega_{3}}\right) p_{\Omega_{1} \Omega_{2} \Omega_{3}}\left(\Omega_{1} \Omega_{2} \Omega_{3}\right)=I_{1}+I_{2} \\
& \quad+I_{3}=2 I_{1}+I_{2} .
\end{aligned}
$$

Functions $F_{x}\left(w / \Omega_{1}\right), F_{y}\left(w / \Omega_{2}\right)$, and $F_{z}\left(w / \Omega_{3}\right)$ are given by (23), (24), and (25), respectively, and $p_{\Omega_{1} \Omega_{2} \Omega_{3}}\left(\Omega_{1} \Omega_{2} \Omega_{3}\right)$ with (13).

Integral $I_{1}$ is equal to

$$
\begin{aligned}
I_{1} & =\frac{1}{\lambda^{2 \mu-1}} \frac{1}{\Gamma(c)\left(1-\rho^{2}\right) \rho^{c-1} \Omega_{0}^{c+2}} \\
& \cdot \sum_{i_{1}=0}^{\infty} \frac{1}{i_{1} ! \Gamma\left(i_{1}+2 \mu\right)} \lambda^{i_{1}+\mu-1 / 2} \sum_{j_{1}=0}^{\infty}\left(\frac{\sqrt{\rho}}{\Omega_{0}(1-\rho)}\right)^{2 j_{1}+c-1} \\
& \cdot \frac{1}{j_{1} ! \Gamma\left(j_{1}+c\right)} \sum_{j_{2}=0}^{\infty}\left(\frac{\sqrt{\rho}}{\Omega_{0}(1-\rho)}\right)^{2 j_{2}+c-1} \frac{1}{j_{2} ! \Gamma\left(j_{2}+c\right)} \\
& \cdot \int_{0}^{\infty} d \Omega_{1} \Omega_{1}^{-1-i_{1}-\mu+1 / 2+i_{1}+2 \mu+j_{1}+c-1} e^{-\lambda / \Omega_{1}-\Omega_{1} / \Omega_{0}(1-\rho)} \gamma\left(i_{1}\right. \\
& \left.+2 \mu, \frac{w^{2}}{\Omega_{1}}\right) \int_{0}^{\Omega_{1}} d \Omega_{2} \Omega_{2}^{j_{1}+j_{2}+c-1} e^{-\Omega_{2}(1+\rho) / \Omega_{0}(1-\rho)} \\
& \cdot \int_{0}^{\Omega_{1}} d \Omega_{3} \Omega_{3}^{j_{2}+c-1} e^{-\Omega_{3} / \Omega_{0}(1-\rho)} .
\end{aligned}
$$

After using [12] for resolving the second and third integrals in (27), $I_{1}$ becomes

$$
\begin{aligned}
I_{1}= & \frac{1}{\lambda^{2 \mu-1}} \frac{1}{\Gamma(c)\left(1-\rho^{2}\right) \rho^{c-1} \Omega_{0}^{c+2}} \\
& \cdot \sum_{i_{1}=0}^{\infty} \frac{1}{i_{1} ! \Gamma\left(i_{1}+2 \mu\right)} \lambda^{i_{1}+\mu-1 / 2} \\
& \cdot \sum_{j_{1}=0}^{\infty}\left(\frac{\sqrt{\rho}}{\Omega_{0}(1-\rho)}\right)^{2 j_{1}+c-1} \frac{1}{j_{1} ! \Gamma\left(j_{1}+c\right)} \\
& \cdot \sum_{j_{2}=0}^{\infty}\left(\frac{\sqrt{\rho}}{\Omega_{0}(1-\rho)}\right)^{2 j_{2}+c-1} \frac{1}{j_{2} ! \Gamma\left(j_{2}+c\right)} \\
& \cdot\left(\frac{\Omega_{0}(1-\rho)}{1+\rho}\right)^{j_{1}+j_{2}+c}\left(\Omega_{0}(1-\rho)\right)^{j_{2}+c} \\
& \cdot \int_{0}^{\infty} \Omega_{1} \Omega_{1}^{-1 / 2+\mu+j_{1}+c-1} e^{-\lambda / \Omega_{1}-\Omega_{1} / \Omega_{0}(1-\rho)} \\
& \cdot \gamma\left(i_{1}+2 \mu, \frac{w^{2}}{\Omega_{1}}\right) \gamma\left(j_{1}+j_{2}+c, \frac{(1+\rho) \Omega_{1}}{\Omega_{0}(1-\rho)}\right) \\
& \cdot \gamma\left(j_{2}+c, \frac{\Omega_{1}}{\Omega_{0}(1-\rho)}\right) .
\end{aligned}
$$

After developing Gamma function defined by (17) and using [12] the following is obtained:

$$
\begin{aligned}
I_{1}= & \frac{1}{\lambda^{2 \mu-1}} \frac{1}{\Gamma(c)\left(1-\rho^{2}\right) \rho^{c-1} \Omega_{0}^{c+2}} \\
& \cdot \sum_{i_{1}=0}^{\infty} \frac{1}{i_{1} ! \Gamma\left(i_{1}+2 \mu\right)} \lambda^{i_{1}+\mu-1 / 2} \sum_{j_{1}=0}^{\infty}\left(\frac{\sqrt{\rho}}{\Omega_{0}(1-\rho)}\right)^{2 j_{1}+c-1} \\
& \cdot \frac{1}{j_{1} ! \Gamma\left(j_{1}+c\right)} \sum_{j_{2}=0}^{\infty}\left(\frac{\sqrt{\rho}}{\Omega_{0}(1-\rho)}\right)^{2 j_{2}+c-1} \frac{1}{j_{2} ! \Gamma\left(j_{2}+c\right)} \\
& \cdot\left(\frac{\Omega_{0}(1-\rho)}{1+\rho}\right)^{j_{1}+j_{2}+c}\left(\Omega_{0}(1-\rho)\right)^{j_{2}+c} \frac{1}{i_{1}+2 \mu} \\
& \cdot w^{2 i_{1}+4 \mu} \sum_{j_{3}=0}^{\infty} \frac{\left(i_{1}+2 \mu\right) !}{\left(i_{1}+2 \mu+j_{3}\right) !} w^{2 j_{3}} \\
& \cdot \frac{1}{j_{1}+j_{2}+c}\left(\frac{(1+\rho)}{\Omega_{0}(1-\rho)}\right)^{j_{1}+j_{2}+c} \\
& \cdot \sum_{j_{4}=0}^{\infty} \frac{\left(j_{1}+j_{2}+c\right) !}{\left(j_{1}+j_{2}+c+j_{4}\right) !}\left(\frac{(1+\rho)}{\Omega_{0}(1-\rho)}\right)^{j_{4}} \frac{1}{j_{2}+c} \\
& \cdot \frac{1}{\left(\Omega_{0}(1-\rho)\right)^{j_{2}+c}} \sum_{j_{5}=0}^{\infty} \frac{\left(j_{2}+c\right) !}{\left(j_{2}+c+j_{5}\right) !} \frac{1}{\left(\Omega_{0}(1-\rho)\right)^{j_{5}}} \\
& \cdot\left(\frac{\left(\lambda+w^{2}\right) \Omega_{0}(1-\rho)}{(3+\rho)}\right)^{-1 / 4-\mu / 2+j_{1}+j_{2}-j_{3} / 2+j_{4} / 2+j_{5} / 2+3 c / 2} \\
& \cdot K_{-1 / 2-\mu-i_{1}+2 j_{1}+2 j_{2}-j_{3}+j_{4}+j_{5}+3 c}\left(2 \sqrt{\frac{\left(\lambda+w^{2}\right)(3+\rho)}{\Omega_{0}(1-\rho)}}\right) .
\end{aligned}
$$


Journal of Electrical and Computer Engineering

7

Integral $I_{2}$ is equal to

$$
\begin{aligned}
I_{2} & =\frac{1}{\lambda^{2 \mu-1}} \frac{1}{\Gamma(c)\left(1-\rho^{2}\right) \rho^{c-1} \Omega_{0}^{c+2}} \sum_{i_{2}=0}^{\infty} \frac{1}{i_{2} ! \Gamma\left(i_{2}+2 \mu\right)} \lambda^{i_{2}+\mu-1 / 2} \\
& \cdot \sum_{j_{1}=0}^{\infty}\left(\frac{\sqrt{\rho}}{\Omega_{0}(1-\rho)}\right)^{2 j_{1}+c-1} \frac{1}{j_{1} ! \Gamma\left(j_{1}+c\right)} \\
& \cdot \sum_{j_{2}=0}^{\infty}\left(\frac{\sqrt{\rho}}{\Omega_{0}(1-\rho)}\right)^{2 j_{2}+c-1} \frac{1}{j_{2} ! \Gamma\left(j_{2}+c\right)} \\
& \cdot \int_{0}^{\infty} d \Omega_{2} \Omega_{2}^{-1-i_{2}-\mu+1 / 2+i_{2}+2 \mu+j_{1}+j_{2}+c-1} e^{-\lambda / \Omega_{2}-\Omega_{2}(1+\rho) / \Omega_{0}(1-\rho)} \gamma\left(i_{2}\right. \\
& \left.+2 \mu, \frac{w^{2}}{\Omega_{2}}\right) \int_{0}^{\Omega_{2}} d \Omega_{1} \Omega_{1}^{j_{1}+c-1} e^{-\Omega_{1} / \Omega_{0}(1-\rho)} \\
& \cdot \int_{0}^{\Omega_{2}} d \Omega_{3} \Omega_{3}^{j_{2}+c-1} e^{-\Omega_{3} / \Omega_{0}(1-\rho)} .
\end{aligned}
$$

After using the procedure for solving $I_{1}, I_{2}$ becomes

$$
\begin{aligned}
I_{2}= & \frac{1}{\lambda^{2 \mu-1}} \frac{1}{\Gamma(c)\left(1-\rho^{2}\right) \rho^{c-1} \Omega_{0}^{c+2}} \sum_{i_{2}=0}^{\infty} \frac{1}{i_{2} ! \Gamma\left(i_{2}+2 \mu\right)} \lambda^{i_{2}+\mu-1 / 2} \\
& \cdot \sum_{j_{1}=0}^{\infty}\left(\frac{\sqrt{\rho}}{\Omega_{0}(1-\rho)}\right)^{2 j_{1}+c-1} \frac{1}{j_{1} ! \Gamma\left(j_{1}+c\right)} \\
& \cdot \sum_{j_{2}=0}^{\infty}\left(\frac{\sqrt{\rho}}{\Omega_{0}(1-\rho)}\right)^{2 j_{2}+c-1} \frac{1}{j_{2} ! \Gamma\left(j_{2}+c\right)}\left(\Omega_{0}(1-\rho)\right)^{j_{1}+j_{2}+2 c} \\
& \cdot \frac{1}{i_{2}+2 \mu} w^{2 i_{2}+4 \mu} \sum_{j_{3}=0}^{\infty} \frac{\left(i_{2}+2 \mu\right) !}{\left(i_{2}+2 \mu+j_{3}\right) !} w^{2 j_{3}} \frac{1}{j_{1}+c} \\
& \cdot \frac{1}{\left(\Omega_{0}(1-\rho)\right)^{j_{1}+c}} \sum_{j_{4}=0}^{\infty} \frac{\left(j_{1}+c\right) !}{\left(j_{1}+c+j_{4}\right) !} \frac{1}{\left(\Omega_{0}(1-\rho)\right)^{j_{4}}} \frac{1}{j_{2}+c} \\
& \cdot \frac{1}{\left(\Omega_{0}(1-\rho)\right)^{j_{2}+c}} \sum_{j_{5}=0}^{\infty} \frac{\left(j_{2}+c\right) !}{\left(j_{2}+c+j_{5}\right) !} \frac{1}{\left(\Omega_{0}(1-\rho)\right)^{j_{5}}} \\
& \cdot\left(\frac{\left(\lambda+w^{2}\right) \Omega_{0}(1-\rho)}{(3+\rho)}\right)^{-1 / 4-\mu / 2-i_{2} / 2+j_{1}+j_{2}-j_{3} / 2+j_{4} / 2+j_{5} / 2+3 c / 2} \\
& \cdot K_{-1 / 2-\mu-i_{2}+2 j_{1}+2 j_{2}-j_{3}+j_{4}+j_{5}+3 c}\left(2 \sqrt{\frac{\left(\lambda+w^{2}\right)(3+\rho)}{\Omega_{0}(1-\rho)}}\right) .
\end{aligned}
$$

After replacing the integrals $I_{1}$ and $I_{2}$ in (26), we obtain an expression for CDF of macrodiversity SC receiver output signal envelope:

$$
\begin{aligned}
& F_{w}(w)=2 \frac{1}{\lambda^{2 \mu-1}} \frac{1}{\Gamma(c)\left(1-\rho^{2}\right) \rho^{c-1} \Omega_{0}^{c+2}} \sum_{i_{1}=0}^{\infty} \frac{\lambda^{i_{1}+\mu-1 / 2}}{i_{1} ! \Gamma\left(i_{1}+2 \mu\right)} \\
& \cdot \sum_{j_{1}=0}^{\infty}\left(\frac{\sqrt{\rho}}{\Omega_{0}(1-\rho)}\right)^{2 j_{1}+c-1} \frac{1}{j_{1} ! \Gamma\left(j_{1}+c\right)} \\
& \cdot \sum_{j_{2}=0}^{\infty}\left(\frac{\sqrt{\rho}}{\Omega_{0}(1-\rho)}\right)^{2 j_{2}+c-1} \frac{1}{j_{2} ! \Gamma\left(j_{2}+c\right)} \frac{w^{2 i_{1}+4 \mu}}{i_{1}+2 \mu}
\end{aligned}
$$

$$
\begin{aligned}
& \cdot \sum_{j_{3}=0}^{\infty} \frac{\left(i_{1}+2 \mu\right) !}{\left(i_{1}+2 \mu+j_{3}\right) !} w^{2 j_{3}}\left[\left(\frac{\Omega_{0}(1-\rho)}{1+\rho}\right)^{j_{1}+2 j_{2}+2 c}\right. \\
& \cdot \frac{1}{j_{1}+j_{2}+c}\left(\frac{(1+\rho)}{\Omega_{0}(1-\rho)}\right)^{j_{1}+j_{2}+c} \\
& \cdot \sum_{j_{4}=0}^{\infty} \frac{\left(j_{1}+j_{2}+c\right) !}{\left(j_{1}+j_{2}+c+j_{4}\right) !}\left(\frac{(1+\rho)}{\Omega_{0}(1-\rho)}\right)^{j_{4}} \frac{1}{j_{2}+c} \\
& \cdot \frac{1}{\left(\Omega_{0}(1-\rho)\right)^{j_{2}+c}} \sum_{j_{5}=0}^{\infty} \frac{\left(j_{2}+c\right) !}{\left(j_{2}+c+j_{5}\right) !} \frac{1}{\left(\Omega_{0}(1-\rho)\right)^{j_{5}}} \\
& \cdot\left(\frac{\left(\lambda+w^{2}\right) \Omega_{0}(1-\rho)}{(3+\rho)}\right)^{-1 / 4-\mu / 2+j_{1}+j_{2}-j_{3} / 2+j_{4} / 2+j_{5} / 2+3 c / 2} \\
& \cdot K_{-1 / 2-\mu-i_{1}+2 j_{1}+2 j_{2}-j_{3}+j_{4}+j_{5}+3 c}\left(2 \sqrt{\frac{\left(\lambda+w^{2}\right)(3+\rho)}{\Omega_{0}(1-\rho)}}\right) \\
& +\left(\Omega_{0}(1-\rho)\right)^{j_{1}+j_{2}+2 c} \frac{1}{j_{1}+c} \frac{1}{\left(\Omega_{0}(1-\rho)\right)^{j_{1}+c}} \\
& \cdot \sum_{j_{4}=0}^{\infty} \frac{\left(j_{1}+c\right) !}{\left(j_{1}+c+j_{4}\right) !} \frac{1}{\left(\Omega_{0}(1-\rho)\right)^{j_{4}}} \frac{1}{j_{2}+c} \frac{1}{\left(\Omega_{0}(1-\rho)\right)^{j_{2}+c}} \\
& \cdot \sum_{j_{5}=0}^{\infty} \frac{\left(j_{2}+c\right) !}{\left(j_{2}+c+j_{5}\right) !} \frac{1}{\left(\Omega_{0}(1-\rho)\right)^{j_{5}}} \\
& \cdot\left(\frac{\left(\lambda+w^{2}\right) \Omega_{0}(1-\rho)}{(3+\rho)}\right)^{-1 / 4-\mu / 2-i_{1} / 2+j_{1}+j_{2}-j_{3} / 2+j_{4} / 2+j_{5} / 2+3 c / 2} \\
& \left.\cdot K_{-1 / 2-\mu-i_{1}+2 j_{1}+2 j_{2}-j_{3}+j_{4}+j_{5}+3 c}\left(2 \sqrt{\frac{\left(\lambda+w^{2}\right)(3+\rho)}{\Omega_{0}(1-\rho)}}\right)\right] .
\end{aligned}
$$

5. Numerical Results

Probability density function of macrodiversity SC receiver output signal envelope versus SC receiver output signal envelope is plotted in Figure 2 for several values of $k$ - $\mu$ fading severity parameter $\mu$.

In Figure 3, cumulative distribution function of macrodiverity SC receiver output signal envelope versus SC receiver output signal envelope is plotted for different $k-\mu$ fading severity parameter $\mu$, Rician factor $k$, Gamma fading severity $c$, and correlation coefficient $\rho$.

In Figure 4, cumulative distribution function of macrodiversity SC receiver output signal envelope versus Rician factor $k$ is plotted for different $k-\mu$ fading severity parameter $\mu$ and correlation coefficient $\rho$.

6. Conclusion

In this paper analysis of diversity system with three microdiversity MRC receivers and one macrodiversity SC receiver was done. At the inputs of microdiversity MRC receivers exist independent $k-\mu$ short term fading and correlated Gamma long term fading. Macrodiversity SC receiver reduces $k-\mu$ 


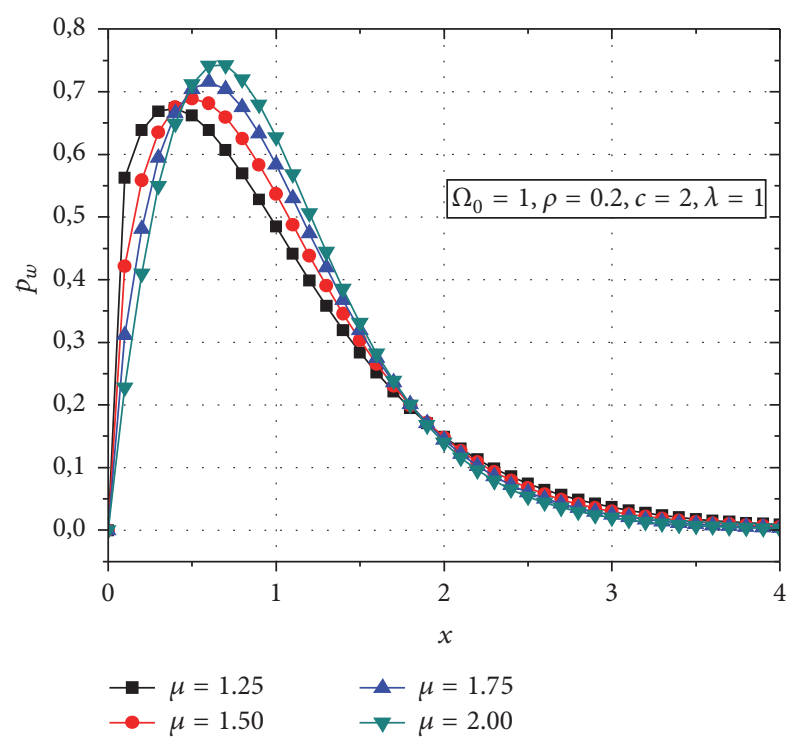

FIGURE 2: Probability density function of microdiversity MRC receiver.

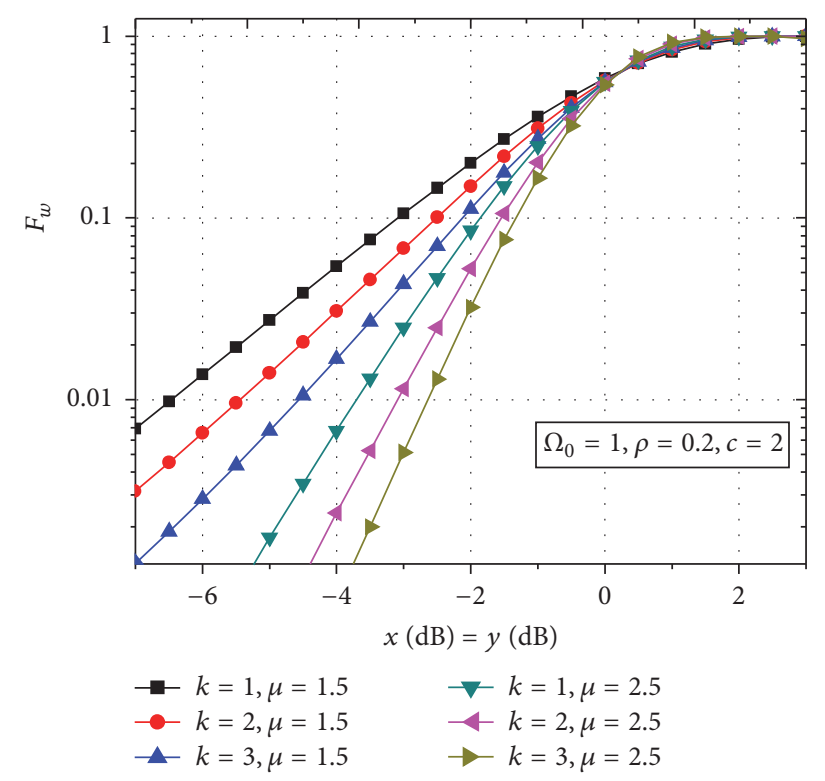

FIGURE 3: Cumulative distribution function of macrodiversity SC receiver.

short term fading effects and microdiversity SC receivers reduce Gamma long term fading effects on system performances. For this system, the probability density function of macrodiversity SC receiver output signal envelope and the cumulative density function of macrodiversity SC receiver output signal envelope was calculated. Probability density function and cumulative density function are very important statistical characteristics. By using them we can calculate outage probability and average bit error probability.

By setting $k=0$, the $k-\mu$ distribution reduces to Nakagami-m distribution and Rician distribution can be

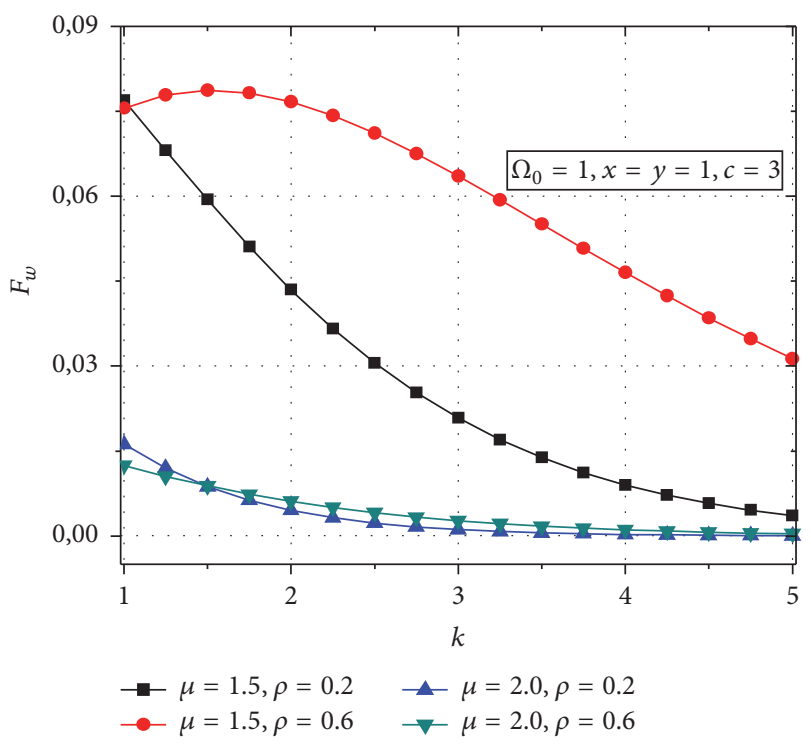

FIGURE 4: Cumulative distribution function of macrodiversity SC receiver output signal envelope versus Rician factor $k$.

derived from $k-\mu$ distribution by setting for $\mu=1$. The $k$ $\mu$ distribution approximates Rayleigh distribution by setting $k=0$ and $\mu=1$. System performances are on low level if we increase fading severity. With less $k$ factor fading becomes of high severity.

Increase of Rician $k$ factor contributes to the decrease of cumulative density function. Cumulative density function of macrodiversity SC receiver output signal envelope decreases significantly faster for lower values of $\mu$ parameters, in comparison to higher value of parameter $\mu$. With the increasing of correlation coefficient, the value of cumulative density function of macrodiversity SC receiver output signal envelope decreases. Signal envelope increases the value of cumulative density function growth and tends to 1 . With increase of Rician $k$ factor and the number of clusters $\mu$, cumulative probability of signal grows faster.

\section{Competing Interests}

The authors declare that they have no competing interests.

\section{References}

[1] G. L. Stuber, Mobile Communication, Kluwer Academic, Dordrecht, The Netherlands, 2nd edition, 2003.

[2] S. Panic, M. Stefanovic, J. Anastasov, and P. Spalevic, Fading and Interference Mitigation in Wireless Communications, CRC Press, Boca Raton, Fla, USA, 2013.

[3] M. K. Simon and M. S. Alouini, Digital Communication over Fading Channels, John Wiley \& Sons, New York, NY, USA, 2000.

[4] M. Milišić, M. Hamza, and M. Hadžialić, "BEP/SEP and outage performance analysis of L-branch maximal-ratio combiner for $\kappa-\mu$ fading," International Journal of Digital Multimedia Broadcasting, vol. 2009, Article ID 573404, 8 pages, 2009.

[5] G. Malmgren, "On the performance of single frequency networks in correlated shadow fading," IEEE Transactions on Broadcasting, vol. 43, no. 2, pp. 155-160, 1997. 
[6] M. D. Yacoub, "The $\eta-\mu$ distribution and the $\kappa-\mu$ distribution," IEEE Antennas and Propagation Magazine, vol. 49, no. 1, pp. 6881, 2007.

[7] J. Proakis, Digital Communications, McGraw-Hill, New York, NY, USA, 4th edition, 2001.

[8] P. M. Shankar, "Analysis of microdiversity and dual channel macrodiversity in shadowed fading channels using a compound fading model," International Journal of Electronics and Communications, vol. 62, no. 6, pp. 445-449, 2008.

[9] J. Zhang and V. Aalo, "Effect of macrodiversity on averageerror probabilities in a Rician fading channel with correlated lognormal shadowing," IEEE Transactions on Communications, vol. 49, no. 1, pp. 14-18, 2001.

[10] D. M. Stefanović, S. R. Panić, and P. Ć. Spalević, "Second-order statistics of SC macrodiversity system operating over Gamma shadowed Nakagami-m fading channels," AEU-International Journal of Electronics and Communications, vol. 65, no. 5, pp. 413-418, 2011.

[11] N. M. Sekulović and M. Č. Stefanović, "Performance analysis of system with micro- and macrodiversity reception in correlated gamma shadowed rician fading channels," Wireless Personal Communications, vol. 65, no. 1, pp. 143-156, 2012.

[12] I. S. Gradshteyn and I. M. Ryzhik, Table of Integrals, Series, and Products, Academic Press, San Diego, Calif, USA, 2000. 


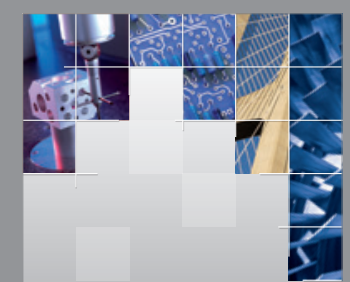

\section{Enfincering}
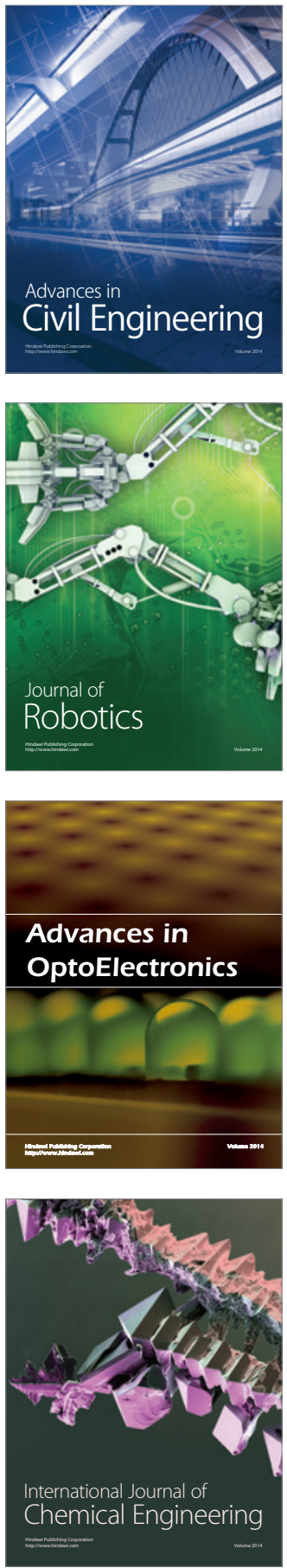

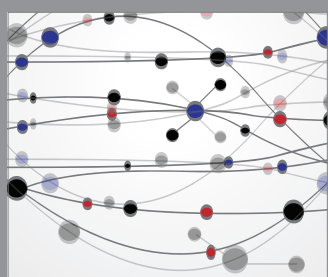

The Scientific World Journal

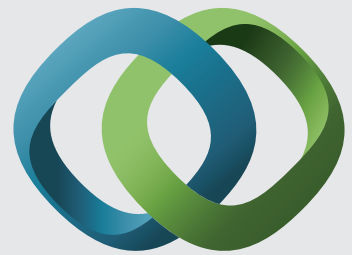

\section{Hindawi}

Submit your manuscripts at

http://www.hindawi.com
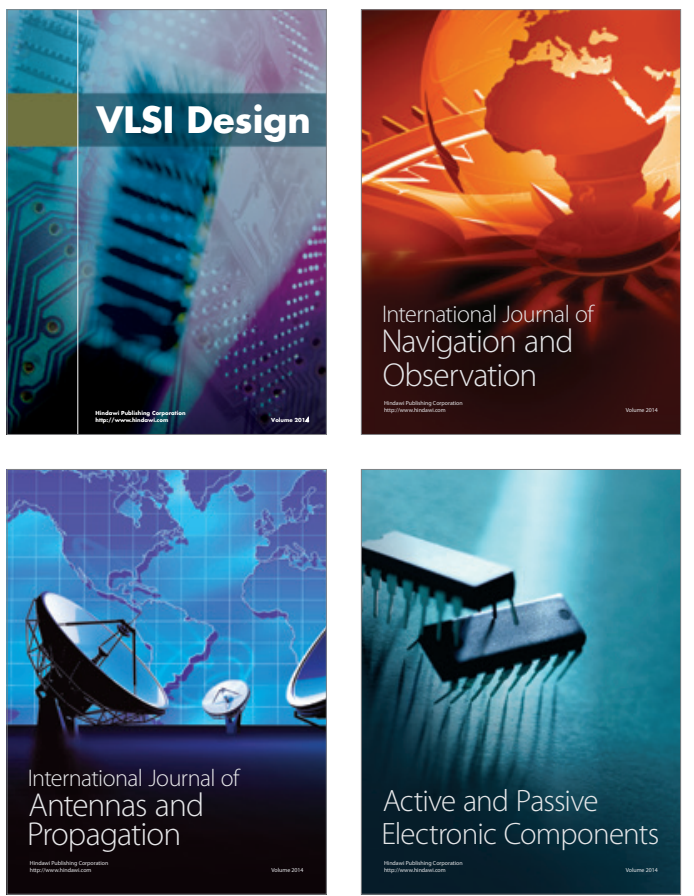
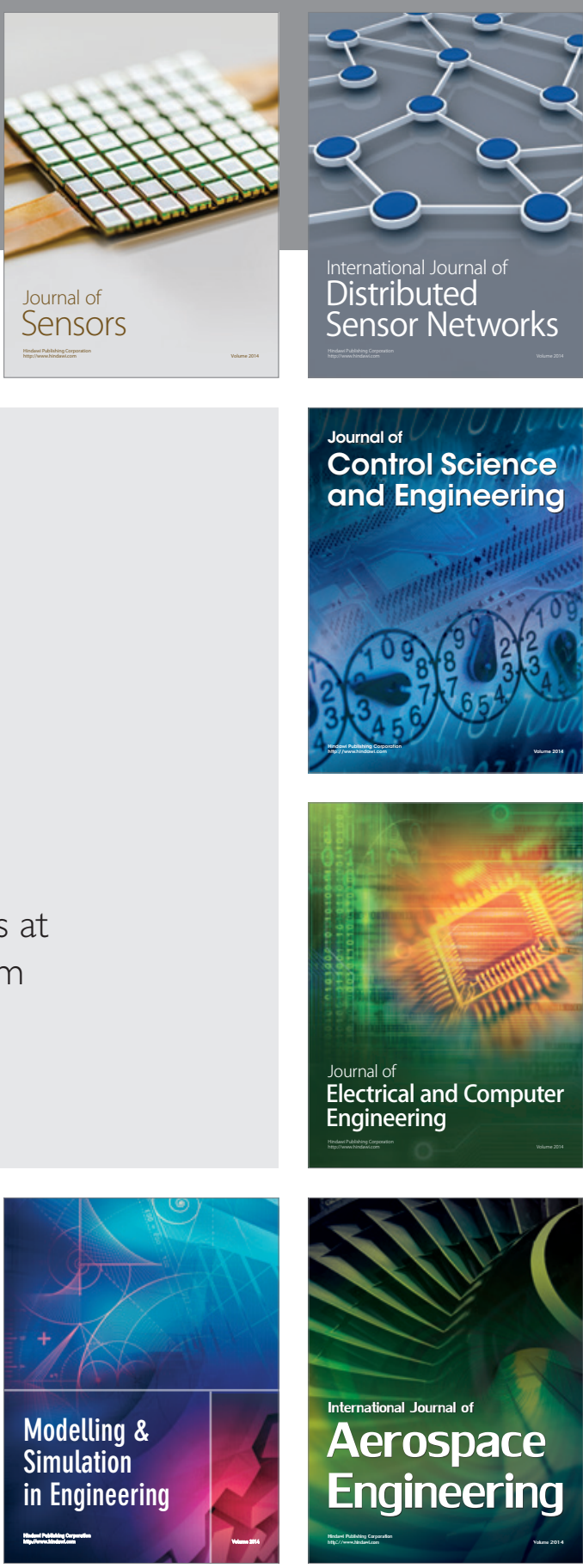

International Journal of

Distributed

Sensor Networks

Journal of

Control Science

and Engineering
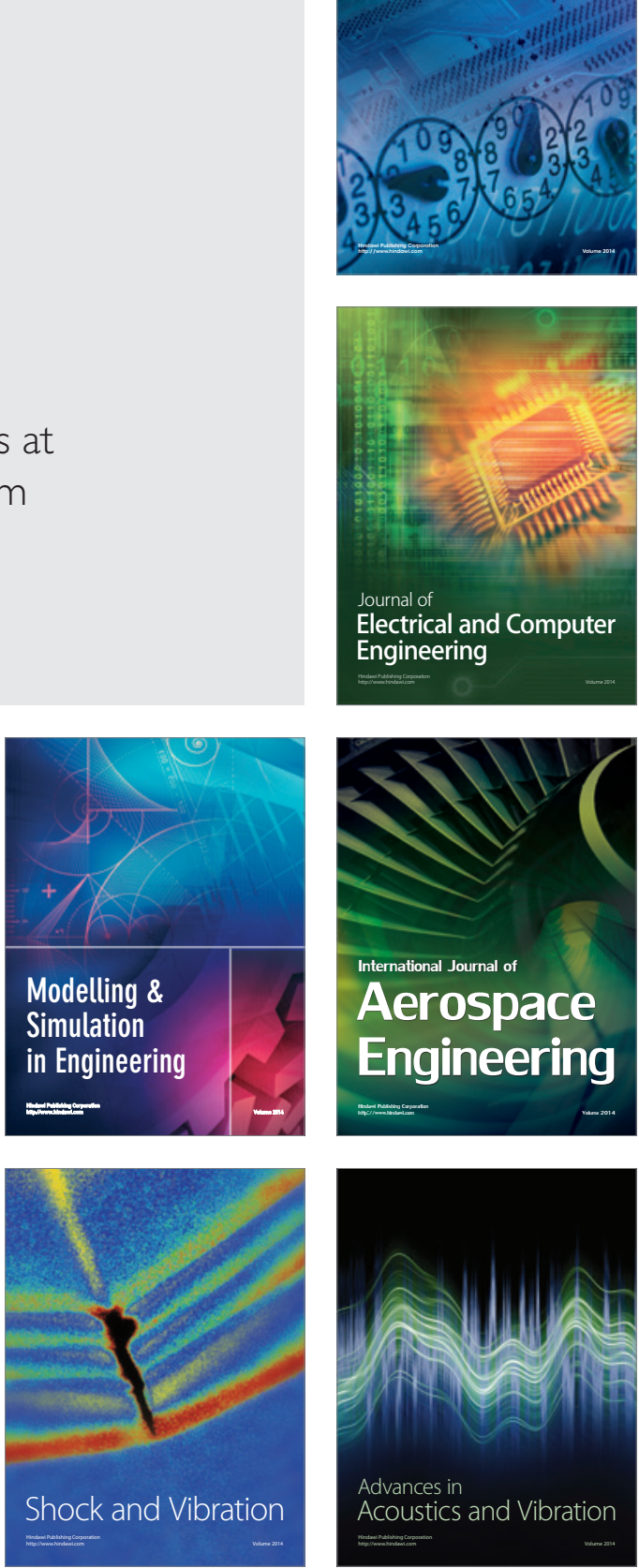